\title{
Critical Dynamics of the Contact Process with Quenched Disorder
}

\author{
Adriana G. Moreira ${ }^{\dagger, a}$ and Ronald Dickman ${ }^{\ddagger, b}$ \\ †Departamento de Física, UFMG, CP 702, CEP 30161-970, Belo Horizonte, Brazil \\ and \\ ${ }^{\ddagger}$ Department of Physics and Astronomy, Lehman College, CUNY, Bronx, NY 10468-1589, USA
}

(October 11, 2018)

\begin{abstract}
We study critical spreading dynamics in the two-dimensional contact process (CP) with quenched disorder in the form of random dilution. In the pure model, spreading from a single particle at the critical point $\lambda_{c}$ is characterized by the critical exponents of directed percolation: in $2+1$ dimensions, $\delta=0.46$, $\eta=0.214$, and $z=1.13$. Disorder causes a dramatic change in the critical exponents, to $\delta \simeq 0.60, \eta \simeq-0.42$, and $z \simeq 0.24$. These exponents govern spreading following a long crossover period. The usual hyperscaling relation, $4 \delta+2 \eta=d z$, is violated. Our results support the conjecture by Bramson, Durrett, and Schonmann [Ann. Prob. 19, 960 (1991)], that in two or more dimensions the disordered CP has only a single phase transition.

PACS numbers: 05.50.+q, 02.50.-r, 05.70.Ln
\end{abstract}


Phase transitions between an absorbing state (one, that is, admitting no further evolution), and an active regime occur in models of autocatalytic chemical reactions, epidemics, and transport in disordered media. Critical phenomena attending absorbing-state transitions show a high degree of universality, that has been characterized rather precisely in studies of the contact process (CP) and of directed percolation (DP) [1] [3]. Since manyparticle systems often incorporate frozen-in randomness, it is natural to investigate its effect on an absorbing-state transition. Some time ago, Noest studied the change in the static critical behavior of DP due to quenched disorder 四. In this work we examine its effect on time-dependent critical phenomena at an absorbing-state transition. We focus on the two-dimensional CP, a simple lattice model of an epidemic [5]. Our prime interest is in the effect of disorder on the spread of the critical process from a seed.

At a critical creation rate, $\lambda_{c}$, the pure $\mathrm{CP}$ exhibits a second-order phase transition characterized by the same critical exponents as DP [1]. The well-known Harris criterion [6.7] states that disorder changes the critical exponents if $d \nu_{\perp} \leq 2$, where $d$ is the dimensionality and $\nu_{\perp}$ is the correlation-length exponent of the pure model. Since $\nu_{\perp} \simeq 0.73$ for DP in $2+1$ dimensions, we expect quenched disorder to be relevant in the CP. Indeed, Noest's simulations of one- and two-dimensional stochastic cellular automata (belonging to the DP class), yielded static critical exponents quite distinct from those of DP, when the models were modified to incorporate quenched randomness 四. A field-theoretic study by Obukhov [8] yielded qualitatively consistent results. Marques studied the effects of dilution on the $\mathrm{CP}$ and related models in a mean-field renormalization group study, obtaining a phase diagram in good agreement with simulation [9].

The effect of disorder on time-dependent critical behavior at an absorbing-state transition has received little attention. The only study we are aware of, by Bramson, Durrett, and Schonmann, showed that the one-dimensional CP in a random environment has a new phase in which the process survives but spreads more slowly than linearly with time, t [10]. (In the pure $\mathrm{CP}$ the radius of the active region grows $\propto t$ for any $\lambda>\lambda_{c}$.)

In this paper we report on extensive simulations of time-dependent critical behavior in 
the two-dimensional DCP. The model is defined as follows. Each site of the square lattice $\mathcal{Z}^{2}$ is either vacant or occupied by a particle. Particles are created at vacant sites at rate $\lambda n / 4$, where $n$ is the number of occupied nearest-neighbors, and are annihilated at unit rate, independent of the surrounding configuration. The order parameter is the particle density $\rho$; it vanishes in the vacuum state, which is absorbing. As $\lambda$ is increased beyond $\lambda_{c}=1.6488(1)$, there is a continuous phase transition from the vacuum to an active steady state. We introduce disorder by randomly removing a fraction $x$ of the sites. That is, for each $(i, j) \in \mathcal{Z}^{2}$ there is an independent random variable $\eta(i, j)$ taking values 0 and 1 with probability $x$ and $1-x$, respectively. The DCP is simply the contact process restricted to sites with $\eta(i, j)=1$; those having $\eta(i, j)=0$ are never occupied. (Thus if exactly $m$ neighbors of a given site have $\eta(i, j)=1$, the creation rate at that site is at most $m \lambda / 4$.) Naturally, $1-x$ must exceed the percolation threshold $p_{c}=0.5927$ for there to be any possibility of an active state, since on finite sets the CP is doomed to extinction.

Following the approach of Grassberger and de la Torre [1], we study a large ensemble of trials, all starting from a configuration very close to the absorbing state: a single particle at the origin. For $\lambda>\lambda_{c}(x)$ there is a nonzero probability that the process survives as $t \rightarrow \infty$; for $\lambda \leq \lambda_{c}(x)$ the process dies with probability 1. Of prime interest are $P(t)$, the survival probability at time $t, n(t)$, the mean number of particles (averaged over all trials, including those that die before time $t$ ), and $R^{2}(t)$, the mean-square distance of particles from the origin. At the critical point of the pure CP, these quantities follow asymptotic power-laws,

$$
\begin{aligned}
P(t) & \propto t^{-\delta} \\
n(t) & \propto t^{\eta} \\
R^{2}(t) & \propto t^{z}
\end{aligned}
$$

The exponents satisfy the hyperscaling relation

$$
4 \delta+2 \eta=d z
$$

in $d \leq 4$ dimensions [面]. For $\lambda<\lambda_{c}, P(t)$ and $n(t)$ decay exponentially, while for $\lambda>\lambda_{c}$, 


$$
P(t) \rightarrow P_{\infty} \sim\left(\lambda-\lambda_{c}\right)^{\beta^{\prime}},
$$

and $n(t) \sim t^{d}$. Thus log-log plots of $P(t), n(t)$ and $R^{2}(t)$ approach straight lines at the critical point, and show a positive or negative curvature in the supercritical or subcritical regimes, respectively. We adopt the working hypothesis that the critical point of the DCP is also characterized by asymptotic power laws.

We studied dilutions $x=0.02,0.05,0.1,0.2,0.3$, and 0.35 . Samples of from $10^{5}$ to $2 \times 10^{6}$ trials were generated for each $\lambda$ value of interest, each trial extending to a maximum time $t_{\max } \leq 7 \times 10^{4}$. (As is usual in this sort of simulation, the time increment associated with an elementary event - creation or annihilation - is $\Delta t=1 / N$, where $N$ is the number of particles.) An independent realization of disorder (the variables $\eta(i, j)$ ), is generated for each trial. Our results for $P(t), n(t)$ and $R^{2}(t)$ (see Fig. 1), allow us to compute the local slopes $\delta(t), \eta(t)$, and $z(t)$, using least-squares fits to the data (in a logarithmic plot), distributed symmetrically about a given $t$ (typically in the interval $[t / 3,3 t]$ ). We estimate the exponents by plotting the local slopes versus $1 / t$ and extrapolating to $1 / t \rightarrow 0$ [11], as in Fig. 4. The critical point $\lambda_{c}(x)$ is determined by the criteria that at long times, log-log plots of $P(t)$ appear straight, and that local-slope plots are free of marked curvature. Our results for the critical point are given in Table I. For small $x, \lambda_{c}(x) \approx \lambda_{c}(0) /(1-x)$, as predicted by mean-field theory [9,12].

Perhaps the most surprising feature of the critical DCP is the population size $n(t)$. In the (pure) critical CP $n(t)$ increases monotonically with time; $\eta$ in Eq. (2) is positive. In the critical DCP $n(t)$ decays at long times; $\eta$ is negative. The critical point evolution of $P, n$, and $R^{2}$, shown for several dilutions in Fig. 1, has a regime characterized by pure CP scaling, followed by a long crossover period leading to a different set of power laws. The apparently disparate results obtained for different dilutions are in fact described by a single function. For $n(t)$, this can be seen by shifting the data (plotted on log-log scales), to superimpose the maxima, which causes all of the points to fall close to a single curve (see Fig. 2). If $\tau(x)$ denotes the time at which $n(t ; x)$ attains its maximum, $n_{\max }(x)$, then we have 


$$
n(t ; x)=n_{\max }(x) \mathcal{N}(\tilde{t})
$$

where $\tilde{t}=t / \tau$ and $\mathcal{N}$ is a scaling function. We regard $\tau$ as the crossover time separating pure $\mathrm{CP}$ scaling (for small $\tilde{t})$ from disordered CP critical behavior $(\tilde{t} \rightarrow \infty)$. To scale the data for $P(t)$ and $R^{2}(t)$ we use the same $\tau(x)$ values as for $n$, and fix dilution-dependent amplitudes $A_{P}(x)$ and $A_{R^{2}}(x)$ by optimizing the data collapse. Thus for the survival probability

$$
P(t ; x)=A_{P}(x) \mathcal{P}(\tilde{t})
$$

and for the mean-square spread,

$$
R^{2}(t ; x)=A_{R^{2}}(x) \mathcal{R}(\tilde{t})
$$

To test these scaling expressions we plot $\tilde{P} \equiv P(t ; x) / A_{P}(x), \tilde{n} \equiv n(t ; x) / n_{\max }(x)$ and $\tilde{R}^{2} \equiv R^{2}(t ; x) / A_{R^{2}}(x)$ versus $\tilde{t}$ in Fig. 2. Data for different dilutions collapse nicely onto a single curve. In Fig. 3 we plot the crossover time $\tau$ and the amplitudes versus $x$. For $x \geq 0.1$ these parameters are well-described by power laws, for example, $\tau \propto x^{-3.2(1)}$. (Figures in parentheses denote uncertainties. The corresponding exponents for $A_{P}, n_{\max }$, and $A_{R^{2}}$ are $1.67(4),-0.56(2)$ and $-3.64(3)$, respectively.) Given the limited range of $x$, there is no reason to attribute any fundamental significance to these power laws. The rapid decrease in $\tau$ with increasing $x$ can be understood by noting that for weak disorder, the process must spread over a certain area before randomness becomes manifest; for small $x$, sizable regions of the lattice look nearly regular. Thus for $x \leq 0.2, n(t)$ and $R^{2}(t)$ have not reached their asymptotic behavior at the end of the trials $\left(t_{\max }=7 \times 10^{4}\right)$. Since $n(t ; x=0.02)$ does not exhibit a maximum on simulation time scales, the data for $x=0.02$ are not included in the scaling analysis.

Given that the approach to asymptotic scaling is more rapid for larger dilutions, we turn first to the data for $x=0.35$ to estimate critical exponents. Fig. 4, for $\eta(t)$, shows that the local slope undergoes a marked change during the evolution. $(z(t)$ also changes over a very wide range.) At long times the local slopes for $x=0.3$ and $x=0.35$ exhibit steady trends, permitting reliable extrapolation of their asymptotic values; for smaller dilutions 
only $\delta$ can be estimated with confidence (see Table I). The scaling plots of Fig. 2 show that the scaling functions $\mathcal{P}, \mathcal{N}$, and $\mathcal{R}$ follow power laws with pure-DP exponents for small $\tilde{t} \equiv t / \tau$, and that for large $\tilde{t}$ they approach power laws with the exponents found in the local-slope analyses.

We also determined the ultimate survival probability exponent $\beta^{\prime}$ (defined in Eq. (5)), for dilutions 0.1 and 0.2 . While $\beta^{\prime}$ is conceptually distinct from the order-parameter exponent $\beta$, defined through $\rho \sim\left(\lambda-\lambda_{c}\right)^{\beta}$, these exponents are equal in the CP and allied models. Stationary-state simulations support the relation $\beta=\beta^{\prime}$ for the DCP as well [12].

Table I reveals the dramatic effect dilution has on the exponents. Comparing the critical exponents for the DCP with those of the pure model, it is evident that the shifts all reflect a diminished ability of the critical process to survive and expand in the presence of disorder. While this accords with intuition, the small $z$ value, and negative $\eta$ are unexpected. Most absorbing-state transitions have $\eta \geq 0$, but this does not appear to be a fast rule, so long as $\eta_{s} \equiv \eta+\delta>0 . \quad\left(\eta_{s}\right.$ governs the population in surviving trials.) Negative values of $\eta$ were also found in a (disorder-free) model with multiple absorbing configurations [13]. Equally puzzling is the massive violation of hyperscaling, Eq. (田). In fact, hyperscaling in the DCP may be incompatible with the simple requirement $\eta_{s}>0$; for $d=2$, Eq. (四) implies $\eta_{s}=z-\delta$, which in turn yields (for $x=0.35$ ), $\eta_{s} \simeq-0.35$. Alternatively, if we take $\eta=-\delta$, (that is, as small as possible without forcing $\eta_{s}<0$ ), Eq (4⿴囗十) yields $z=\delta$, in marked disagreement with the data.

One might regard violation of hyperscaling as evidence that our simulations have yet to probe the asymptotic scaling regime. While simulations which run to a finite time cannot rule this out, the data suggest no trend that would bring the exponents into agreement with hyperscaling. Another interpretation [14] is that sample-to-sample fluctuations and lack of self-averaging lead to a violation of the scaling hypothesis itself (as set out, e.g., in Ref. [1]). We hope to resolve this issue in a future study.

Bramson, Durrett and Schonmann studied a one-dimensional CP with disorder in the form of a death rate randomly taking one of two values (independently) at each site [10. 
They demonstrated that this model possesses an intermediate phase in which survival (starting, e.g., from a single particle) is possible, but the active region grows more slowly than linearly. In two or more dimensions, Bramson et al. conjectured, there is no intermediate phase. Our results support this conjecture. Simulations at $x=0.1$, with $\lambda$ close to, but slightly above $\lambda_{c}$ (to be precise, $\lambda=1.86$ and 1.87 , corresponding to $\left(\lambda-\lambda_{c}\right) / \lambda_{c}=0.008$ and 0.014 , respectively), showed $n(t) \sim t^{2}$ (and similarly for $R^{2}(t)$ ), consistent with the radius of the active region growing $\sim t$. Thus a sublinear growth phase, if it exists at all, is confined to a very narrow range of creation rates. While our model incorporates dilution rather than a random death rate, one would expect such an intermediate phase to be a rather general feature of disordered contact processes, so that its apparent absence here argues for the validity of the conjecture. 


\section{TABLES}

TABLE I. Critical parameters and exponents obtained through simulations of the DCP. The numbers in brackets indicate uncertainties.

\begin{tabular}{|c|c|c|r|r|r|}
\hline \hline$x$ & $\lambda_{c}$ & $\beta^{\prime}$ & $\delta$ & $\eta$ & $z$ \\
\hline \hline 0 & $1.6488[1]$ & $0.586[14]$ & $0.460[6]$ & $0.214[8]$ & $1.134[4]$ \\
0.02 & $1.6850[3]$ & $0.566[7]$ & $0.467[1]$ & $0.216[3]$ & $1.104[2]$ \\
0.05 & $1.7405[3]$ & & $0.59[1]$ & & \\
0.1 & $1.8448[4]$ & $0.70[1]$ & $0.59[1]$ & \\
0.2 & $2.097[2]$ & $0.72[2]$ & $0.60[2]$ & & \\
0.3 & $2.437[2]$ & & $0.59[1]$ & $-0.43[3]$ & \\
0.35 & $2.655[2]$ & & $0.59[1]$ & $-0.41[3]$ & $0.27[3]$ \\
\hline \hline
\end{tabular}


In summary, we find that quenched disorder induces a radical change in the critical exponents describing spreading in the contact process. As the population spreads in the critical process, there is a crossover from pure $\mathrm{CP}$ behavior to a new set of critical exponents. The crossover time appears to diverge as the dilution tends to zero. A related finding is the demonstration by Noest of anomalously slow dynamics, in the form of power-law relaxation to the vacuum, in disordered directed percolation [15]. Although our results on scaling are restricted to dilutions $0.05 \leq x \leq 0.35$, we expect that such a crossover occurs for all $0<x<1-p_{c}$, albeit at very long times for small $x$. One is inclined to suppose that the DCP is but one member of a universality class encompassing all disordered models with a continuous transition to a unique absorbing configuration. In support of this we observe that $\delta$ (the only exponent we are able to determine over a wide range of $x$ values), is insensitive to the degree of dilution, and that evolutions for various $x$, with very different crossover times, are described by common scaling functions. Studies of absorbing state transitions in other disordered models are needed to verify the universality hypothesis. Finally, we note that our result, $\beta=\beta^{\prime}=0.71(2)$, is incompatible with that reported by Noest $(\beta=1.10(5))$ for disordered DP in $2+1$ dimensions [ [ [ W]. We shall return to this question in a future work on the static behavior of the diluted contact process.

\section{Acknowledgments}

We thank Dani ben-Avraham for extensive discussions and Geoff Grinstein for helpful suggestions. A.G.M. was supported by CNPq (Brazil). She thanks the staff of Lehman College, City University of New York, for their hospitality during her visit.

${ }^{a}$ e-mail address: dri@fisica.ufmg.br

${ }^{b}$ e-mail address: dickman@lcvax.lehman.cuny.edu 


\section{REFERENCES}

[1] P. Grassberger and A. de la Torre, Ann. Phys. (N.Y.) 122, 373 (1979).

[2] W. Kinzel, Z. Phys. B58, 229 (1985).

[3] R. Dickman, Int. J. Mod. Phys. C4, 271 (1993).

[4] A. J. Noest, Phys. Rev. Lett. 57, 90 (1986).

[5] T. E. Harris, Ann. of Prob. 2, 969 (1974).

[6] A. B. Harris, J. Phys. C 7, 1671 (1974).

[7] A. N. Berker, Physica A 194, 72 (1993).

[8] S. P. Obukhov, JEPT Lett. 45, 172 (1987).

[9] M.C. Marques, J. Phys. A: Math. Gen. 23, 3389 (1990).

[10] M. Bramson, R. Durrett, and R.H. Schonmann, Ann. Prob. 19 960, (1991).

[11] P. Grassberger, J. Phys. A: Math. Gen. 22, 3673 (1989).

[12] A.G. Moreira and R. Dickman, in preparation.

[13] R. Dickman, Phys. Rev. E53, 2223 (1996).

[14] D. ben-Avraham, I. Webman, and I. Kanter, in preparation.

[15] A. J. Noest, Phys. Rev. B 38, 2715 (1988). 


\section{Figure Captions}

FIG. 1. Survival probability $P$, mean population $n$, and mean-square distance of particles from the origin $R^{2}$, in the critical contact process with dilution $x . \diamond: x=0.05 ;+: x=0.2$; $\times: x=0.35$.

FIG. 2. Scaling plots of spreading in the DCP. Upper panel: $\ln \tilde{P}$ vs $\ln \tilde{t}$; the straight lines have slopes of -0.46 and -0.60 . Middle: $\ln \tilde{n}$; the slopes are 0.241 and -0.42 . Bottom: $\ln \tilde{R}^{2}$; the slopes are 1.134 and 0.24. Symbols: $\diamond: x=0.05 ; \square: x=0.10 ;+: x=0.20 ; \triangle: x=0.30$; $\times: x=0.35$.

FIG. 3. Log-log plots of $\tau(\diamond), n_{\max }(\square), A_{P}(+)$, and $A_{R^{2}}(\times)$ vs dilution $x$.

FIG. 4. Local slope $\eta(t)$ versus $t^{-1}$ in the diluted contact process with $x=0.35$. Symbols: $+: \lambda=2.650 ; \times: \lambda=2.655 ; \diamond: \lambda=2.660$. 